\title{
One-dimensional, portable optical coherence tomography system
}

\author{
Aleksandra Kamińska, ${ }^{*}$ and Marcin Strąkowski \\ Faculty of Electronics, Telecommunications and Informatics, Gdańsk University of Technology, \\ 11/12 G. Narutowicza Str., 80-233 Gdańsk, POLAND
}

Received June 08, 2018; accepted June 23, 2018; published June 30, 2018

\begin{abstract}
Imaging methods are an expanding branch of technology, which involves data acquisition and analysis of images for research and diagnostics. This paper has been devoted to the description of the optical design for a one-dimensional, portable optical coherence tomography (OCT) system. The Mach-Zehnder interferometer has been designed in the presented study. The description of the construction and applied hardware solutions have been presented. Moreover, dedicated software tools for signal processing and data acquisition have been developed.
\end{abstract}

Imaging methods are commonly used in many fields of science and industry [1]. Developing new research areas creates new fields of application for precise imaging techniques.

Optical coherence tomography (OCT) is a well-known, high-resolution method of examining the internal structure of tested samples [2]. This technique can be used, for example, for finding defects in materials or for evaluation of smoothness of the layers or coatings [3, 4]. Inline quality control during the materials production process is very important. Due to those needs, an optical coherence tomography system which would be able to work on a production line, has been designed, built and investigated. The project assumes the realization of a compact design. To avoid the use of complicated detection systems like spectrometers, the swept-source OCT (SS-OCT) configuration has been chosen [5].

The design was based on a Mach-Zehnder interferometer. In order to ensure system stability, widely available single-mode fibers (SMF-28e) have been used. The scheme of the OCT system is presented in Fig. 1.

\footnotetext{
*E-mail: aleksandra.kaminska@pg.edu.pl
}

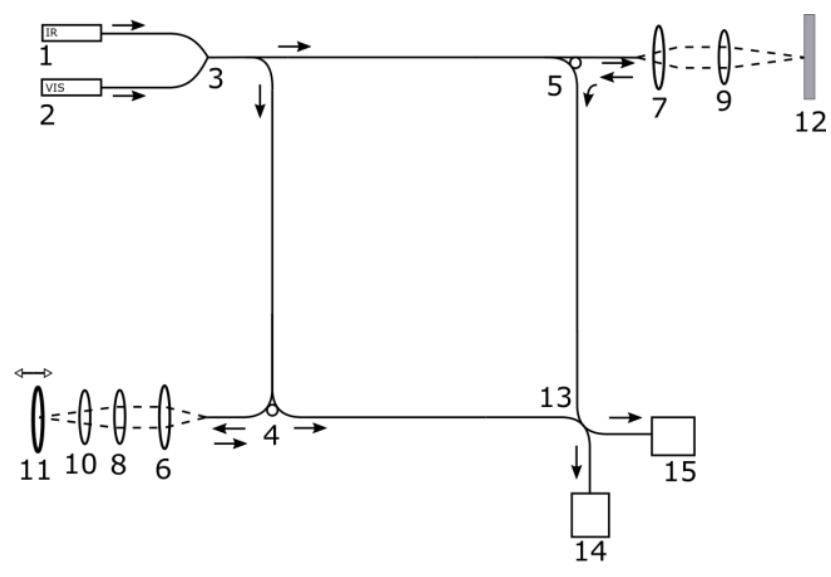

Fig. 1. Scheme of the OCT system built of a swept-source (1), visible laser (2), input beamsplitter (3), circulator in reference arm (4), circulator in tested arm (5), collimators $(6,7)$, focusing lenses $(8,9)$, variable neutral density filter (10), mirror (11), tested sample (12), output coupler (13) and photodiodes $(14,15)$

The spectra range of the used optical light source (HSL2000-WR, Santec) is from $1240 \mathrm{~nm}$ to $1380 \mathrm{~nm}$. Additionally, the system enables connecting a visible light source for monitoring the beam on the sample. For splitting and recombining optical beams, a splitter and a fiber coupler with the coupling ratio 50:50 have been used. Two circulators and focusing optics direct the beam at the mirror and the sample. Additionally, a variable neutral density filter has been placed in the reference arm for the adjustment of optical power. The signal measurement is performed by the balanced photodetector (Thorlabs, PDB450C), which enables signal detection from the interferometer system. It operates by measuring the differential voltage signal from two photodiodes. Additionally, the device allows the signals from each photodiode to be acquired through the output ports. To ensure sample movement under the scanning probe an electromechanical system was designed. The project uses a DC motor that drives the gear. The device uses two mechanical gears to which a one-sided toothed belt was applied. 


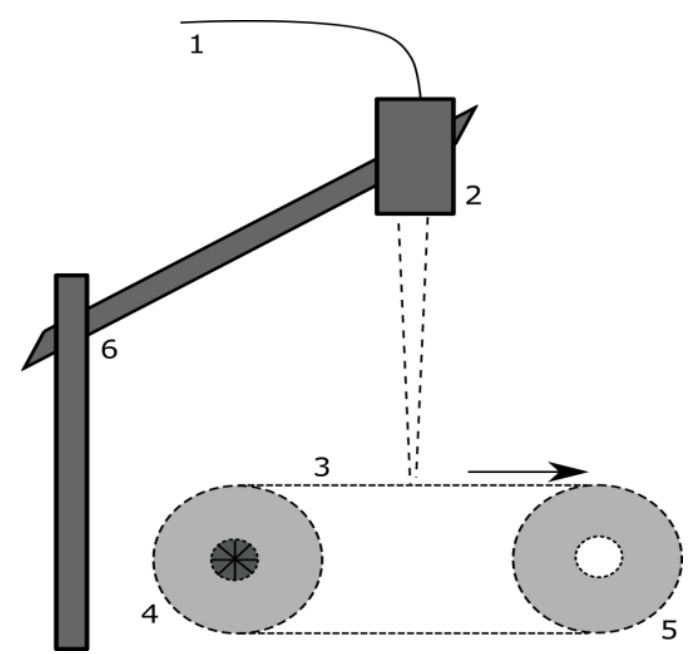

Fig. 2. Scheme of measuring system built of fiber (1), scanning head containing FC/APC connector, collimator and focusing lens (2), belt (3), power gear with a motor (4), gear (5) and structural elements (6).

A polyurethane belt with a trapezoidal tooth profile and a metric pitch T5 was chosen. This material was chosen because of its guaranteed resistance to abrasion. The incorporation of steel cords inside the belt additionally increases endurance [6]. These cords cause the belt to be non-stretchy and accordingly stiff. The flat side of the belt forms the surface on which the materials to be tested will be placed. The width of the belt and its length are equal to $16 \mathrm{~nm}$ and $250 \mathrm{~mm}$, respectively. This approach suits the production lines and avoids the use of large scanning heads.

Data acquisition was carried out using the NI PCI-5922 card, which is compatible with the LabView programming environment. Signal processing began with oversampling [6]. Usually, two to four times up-sampling is acceptable. For this purpose, the zero padding method was used, which is performed in the following steps:

- defining the Fourier transform (FFT algorithm) of the original signal,

- addition of zero values at the end of the signal,

- performing a reverse Fourier transform (IFFT algorithm).

Two times up-sampling has been used and as a result of this operation, the sequence of the samples is doubled.

The next step is the domain change into k-space, which requires signal interpolation to be made.

The relationship between the wavelength and the wave number $k$ for the swept source for $N$ samples is described by Eq. (1).

$$
k_{i}=2 \pi\left(\frac{1}{\lambda_{\max }}+\frac{i}{N-1}\left(\frac{1}{\lambda_{\min }}-\frac{1}{\lambda_{\max }}\right)\right)
$$

where $\lambda_{\min }$ and $\lambda_{\max }$ are the minimum and maximum wavelength respectively, and $i$ is the sample index.
To obtain a linearly sampled spectrum in k-space, it is required to generate interpolation sampling points. The vector $S$ of the indexes is described by Eq. (2).

$$
S=\frac{N-1}{\lambda_{\max }-\lambda_{\min }} \frac{2 \pi}{k_{i}}-\lambda_{\min }
$$

Finally, the target graph (A-scan) was obtained by performing a Fourier transform. Phases of signal processing have been presented in Fig. 3.

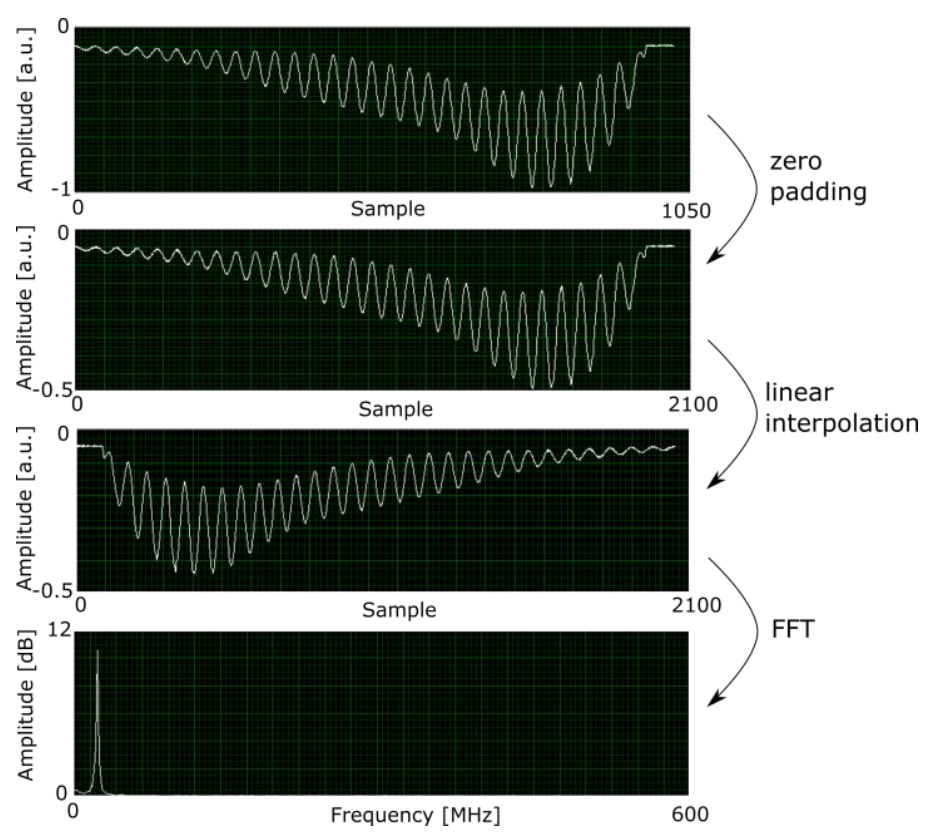

Fig. 3. Digital signal processing: a) acquisition of original signal b) oversampling c) linear interpolation d) FFT (a.u. - arbitrary units).

Based on processed data, OCT images have been generated. Fig. 4 presents the smooth belt, used as a base for samples.

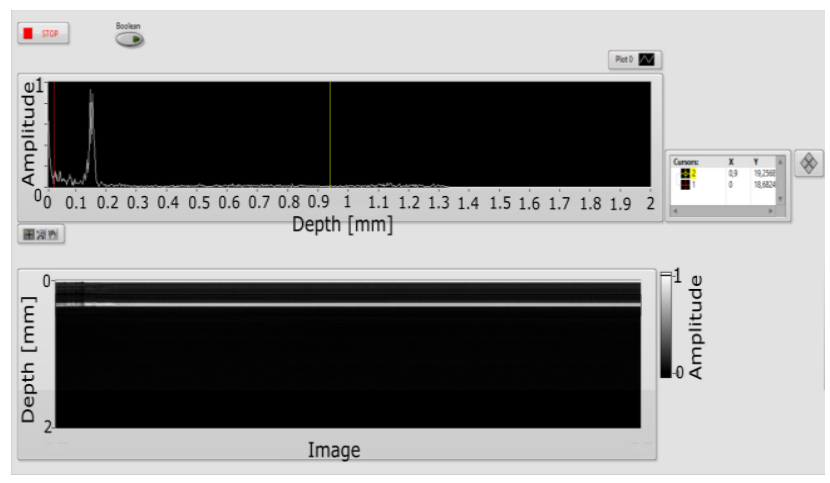

Fig. 4. Single A-scan (above) and OCT image (below) of polyurethane belt. Normalized amplitude corresponds with pixel brightness on the image and the frequency scale (obtained in FFT) can be used to map data to depth profiles. 
To calibrate the graph and OCT image, signal dynamics and maximum scanning depth have been calculated. Moreover, basic parameters of OCT system have been computed [8]. The parameters have been summarized in Tab. 1.

Tabele 1. Parameters of OCT system.

\begin{tabular}{|l|l|}
\hline Parameter & Value \\
\hline Scanning beam diameter & $4.05 \mathrm{~mm}$ \\
\hline Lateral resolution & $5.393 \mu \mathrm{m}$ \\
\hline Axial resolution & $7.58 \mu \mathrm{m}$ \\
\hline Maximum scanning depth & $3.1 \mathrm{~mm}$ \\
\hline Signal dynamics & $40 \mathrm{~dB}$ \\
\hline
\end{tabular}

Dynamics was determined as the difference between the highest and the smallest measurable value of the signal. As the maximum scan depth, the point at which the amplitude drops by $10 \mathrm{~dB}$ is taken.

The designed OCT system is in line with the assumptions. The system is portable and small, which allows working in many places. Moreover, the design can be easily modified and adapted to application needs. The exchangeable optics in the scanning head allows choosing the depth of scanning while changing the light source allows selecting the required system resolution. Figure 5 presents a photo of the OCT system.

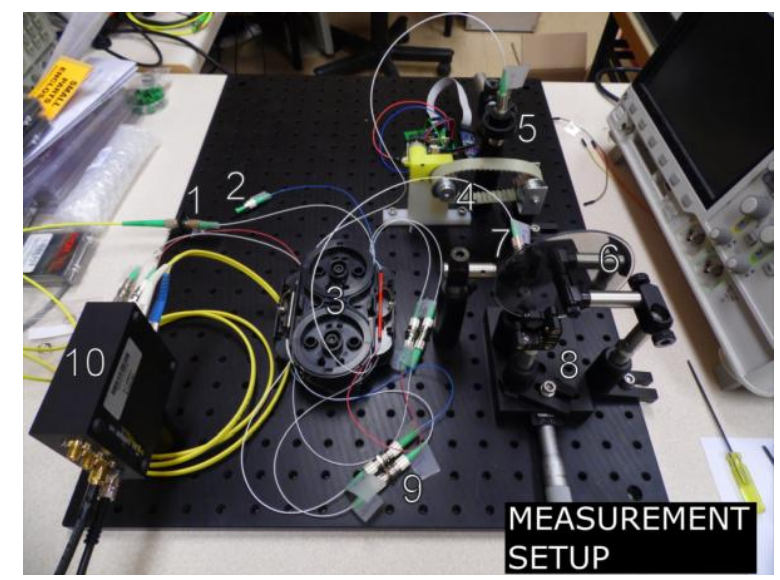

Fig. 5. Design of OCT system: 1 - fiber optic input for a light source, 2 - additional fiber optic input for the visible light source, 3 - fiber optic component trays, 4 - belt in measurement arm, 5 - scanning head, 6 adjustable neutral density filter, 7 - reference arm, 8 - linear stage, 9 FC/APC fiber connectors, 10 - balanced detector.
This research work has been supported by The National Centre for Research and Development (NCBR), Poland under grant no. LIDER/32/205/L-3/11 and DS program of Faculty of Electronics, Telecommunications and Informatics, Gdańsk University of Technology.

\section{References}

[1] M.R. Strąkowski, J. Pluciński, M. Jędrzejewska-Szczerska, R. Hypszer, M. Maciejewski, B.B. Kosmowski, Sensors and Actuators A: Phys. 142(1), 104 (2008).

[2] J.G. Fujimoto, W. Drexler Optical Coherence Tomography: Technology and Applications (Biological and Medical Physics, Biomedical Engineering, New York, Springer 2008).

[3] M. Mujat, R.C. Chan, B. Cense, B.H. Park, C. Joo, T. Akkin, J.F. De Boer, Opt. Expr. 13, 9480 (2005).

[4] H. Liang, M.G. Cid, R.G. Cucu, G.M. Dobre, A.G. Podoleanu, J. Pedro, D. Saunders, Opt. Expr. 13, 6133 (2005).

[5] M.A. Choma, M.V. Sarunic, C. Yang, J.A. Izatt, Opt. Expr. 11, 2183 (2003).

[6] T. Koyama, K.M. Marshek, Mechanism and Machine Theory 23(3), 227 (1988).

[7] M. Ali, R. Parlapalli, Texas Instruments, SPRABB9-June (2010).

[8] B.E.A. Saleh, M.C. Teich, Fundamentals of Photonics, $2^{\text {nd }}$ Edition (New York, Wiley 1991). 